\title{
Automatic Logo EXTRACTION From DOCUMENT IMAGES
}

\author{
Umesh D. Dixit ${ }^{1}$ and M. S. Shirdhonkar ${ }^{2}$ \\ ${ }^{1}$ Department of Electronics \& Communication Engineering, B.L.D.E.A’s CET, Bijapur. \\ ${ }^{2}$ Department of Computer Science and Engineering, B.L.D.E.A’s CET, Bijapur.
}

\begin{abstract}
Logo extraction plays an important role in logo based document image retrieval. Here we present a method for automatic logo extraction from the document images that works for scanned documents containing a logo. Proposed method uses morphological operations for logo extraction. It supports extraction of a logo with its gray level and color information
\end{abstract}

\section{KEYWORDS}

Document image, Preprocessing, Logo extraction

\section{INTRODUCTION}

Logo is a graphic mark or emblem commonly used by commercial enterprises, organizations and even individuals to aid and promote instant public recognition. Logos consists of only graphic symbols or graphics and text indicating name of the organization. The logos can be classified as text logo, graphical logo and mixed logos. A text logo comprises only text information, graphical logos contain some symbols or graphics and a mixed logo includes both text and graphics. The Figure 1 shows examples of these types of logos from University of Maryland (UMD) logo database [1].

\section{Microsoft}

(a) Text logo

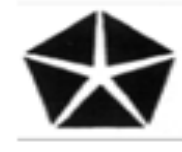

(b) Graphic logo

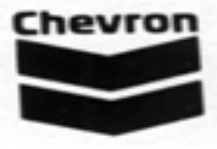

(c) Mixed logo

Figure 1. Types of logos from UMD logo database

The logo based document image retrieval has created a great interest among the research community of document image processing. Logo extraction includes recognizing, detecting and extracting a logo component from document image. However, automatic logo extraction is a challenging task to implement logo based document image retrieval. This paper presents a relatively faster method for automatic logo extraction from scanned documents. 
The rest of the paper is orgainized as follows: Section 2 of this paper describes the related work, Section 3 explains the proposed method, section 4 discuss about results and observations and finally section 5 concludes the paper.

\section{RELATED WORK}

In 1997, Seiden et. al [2] presented logo detection system based on segmentation of the document image into smaller images using a top-down XY cut algorithm. In this approach they used connected components with sixteen features and then applied a rule based strategy for detection of a logo component. In 2001, Hsieh and Fan [3] proposed a technique based on region growing that segments the given input image into possible logo candidates. A logo from these candidates is identified based on color, shape and location. The method proposed in [4]_works on feature selection principles to segment a logo. It selects suitable features such as area, deviation, symmetry, centralization, complexity and 2-level contour representation strings automatically for logo detection from document image.

In 2003, Pham [5] presented a method for logo detection with an assumption that the spatial density of foreground pixels in a logo region will be more than that of non-logo regions. This method first converts the document into a binary form consisting of foreground and background pixels. Spatial density is then computed for each fixed size window (region) and the region that has highest spatial density is recognized as a logo region. In 2006, Hung et. al [6] proposed a shape-based similarity retrieval system based on database classification exploiting the contour and interior region of a shape efficiently. Angular radial transform (ART) region feature is used to compare the query with the candidate sets according to the priority order.

In 2007, Zhu and Doermann [7] employed multi-scale boosting strategy for logo detection and extraction from document image. At coarse image scale they applied two-class Fisher classifier for each connected component. A cascade of simple classifier is then employed at finer image scale to find out a logo from possible set of logo candidates.

In 2009, Wang and Chen presented [8] logo detection method based using boundary extension of feature rectangle. This method works on assumption that logos have white background and is independent of shape of logos. Decision tree is used to increase logo detection rate. It reduced the false positive from the logo candidate pool. Zhu and Doermann [9] proposed a logo-based document image retrieval system that uses a document containing logo as a query. In logo based document retrieval they used detected and segmented a logo by boosting a cascade of classifiers across multiple image scales.

In 2010, Shirdhonkar and Kokare [10] employed discrete wavelet transform (DWT) for automatic logo detection and extraction from the document images. This method computes spatial density using the mean of the energy and standard deviation of the wavelet coefficients for extraction of a $\log$.

In 2011, Hassanzadeh and Pourghassem [11] proposed a frame work for logo detection and recognition based on spatial and structural features. It considers some specifications of logo such as centroid coordinates and intersection of each logo's separated part in detection process. Separated parts of logos are merged using morphological dilation operation. A new feature based on histogram of object occurrence in a logo image is used for recognition purpose.

In 2013, Jha et. al [12] proposed a method for watermark (logo) extraction from distorted watermarked images. Combined discrete wavelet trans- form (DWT) and dynamic stochastic 
resonance (DSR) are used in this method. A logo which is hided in the distorted watermarked image is extracted in this work.

In 2014, Jha et. al [13] presented a technique that employs a dynamic stochastic resonance (DSR) for blind watermark extraction in discrete cosine transform (DCT) domain. DSR is an iterative process that tunes the coefficients of the possibly attacked watermarked image so that the effect of noise is suppressed and hidden information is enhanced.

This paper presents a simple logo extraction method using morphological close and median filtering operations.

\section{Proposed Methodology}

The Figure 2 depicts steps used for logo extraction from document image. In this method we assume non-logo part of the document as noise and we remove the non-logo part of the document image with the help of morphological close operation and then apply median filtering. The different steps involved in logo extraction are gray-scale conversion, image binarization, Morphological close, Median filtering, Logo detection and extraction from original document. These steps are briefly explained below.

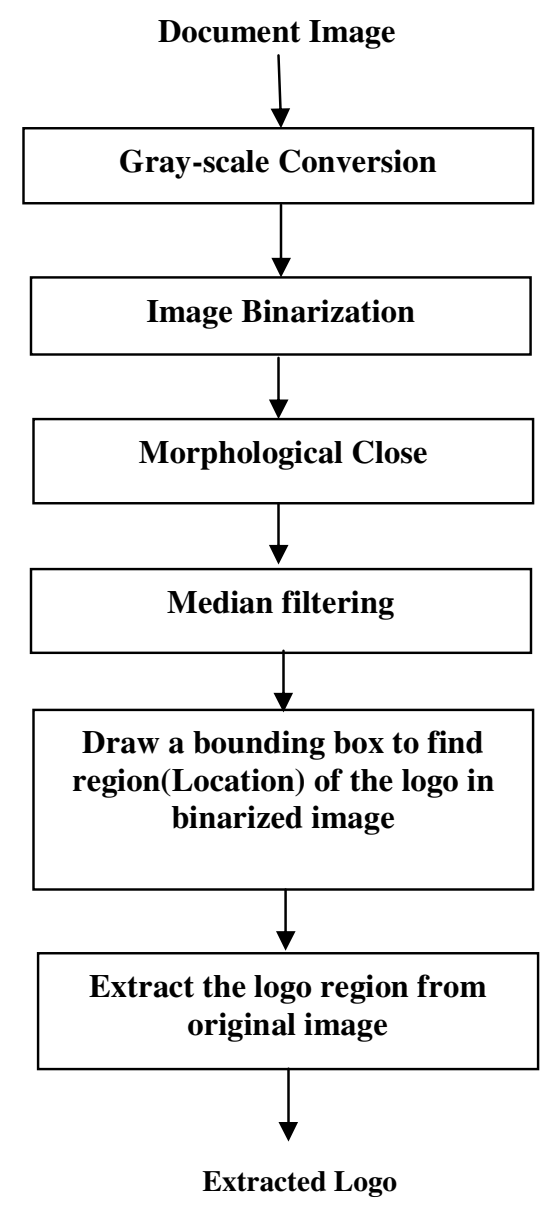

Figure 2. Flowchart of proposed method 
- Gray scale conversion and Binarization: The document image is captured using a scanner. The scanned image of a document may be a color image or gray scale image. If the image scanned is color image, it is first converted into gray level and then converted into binary image using threshold level.

$$
g(x, y)=\left\{\begin{array}{r}
0 \text { if } f(x, y)<T \\
1 \text { if } f(x, y)>=T
\end{array}\right.
$$

Where $f(x, y)$ is gray scale image, $g(x, y)$ is binarized image and ' $T$ ' is appropriate threshold level used for binarization.

- Morphological Closing: Morphological closing of set A by structuring element B is denoted as A $\bullet \mathrm{B}$ and is given by equation (1).

$$
\mathrm{A} \bullet \mathrm{B}=(\mathrm{A} \oplus \mathrm{B}) \Theta \mathrm{B}
$$

In this step we apply morphological closing operation to a binarized image. We use square with size 10 as the structuring element. The size of the structure element square is chosen as 10 to convert characters and other small shapes with foreground color (black) in the document image to background color (white).

- Noise removal: This step applies median filter to remove non-logo part in the document. Mainly it removes impulse noise from the image left after morphological close operation. It keeps much of the logo part as it is in the document and converts remaining part of the document into background color.

- Logo detection and extraction: Now we draw a bounding box to the output obtained from the previous steps. This helps to find out location of a logo in the binarized image. The coordinate points of the bounding box in the binarized image are used to extract a logo from original gray scale/color image.

\section{RESUltS AND Discussion}

The method is tested for more than 50 logos from UMD logo database [1]. Testing is carried out by inserting logos from the in scanned documents. Figure 3 shows some sample logos used for testing and Figure 4 shows results of logo extraction. 


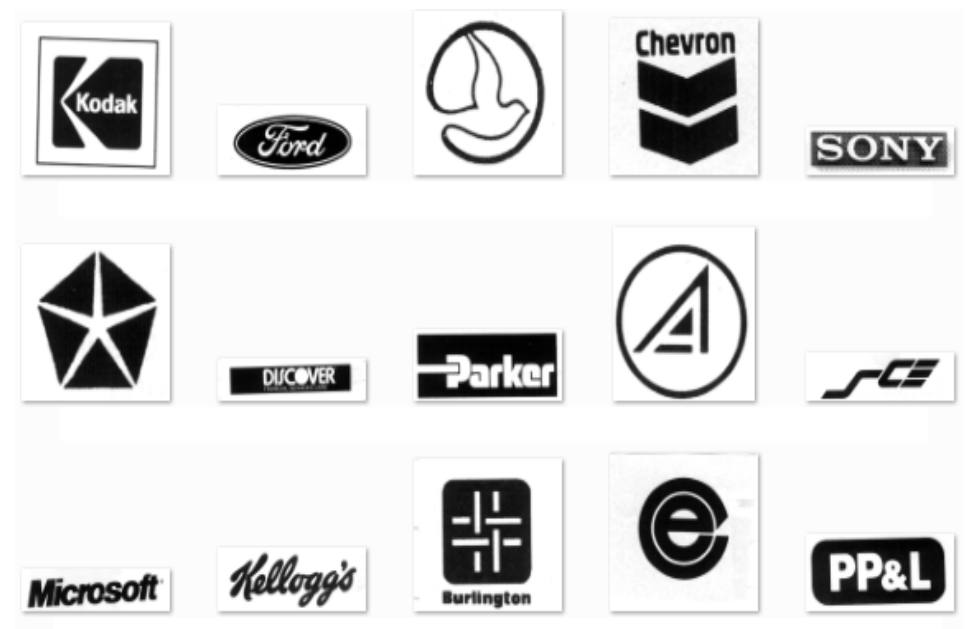

Figure 3. Sample logos used for testing

For testing of the proposed method, we considered two cases:

- Test 1: Here the method is tested for 50 documents each containing a different logo. The algorithm was able to extract logos from all scanned documents.

- Test 2: The same algorithm is also tested by inserting logos at various locations in the document. We found that the proposed algorithm successfully extracts the logos irrespective of its location in the document.

Limitations: It is found that proposed method sometimes makes false detection if the document contains spatially dense components. This limitation can be overcome by further improvement in preprocessing and a suitable classifier.

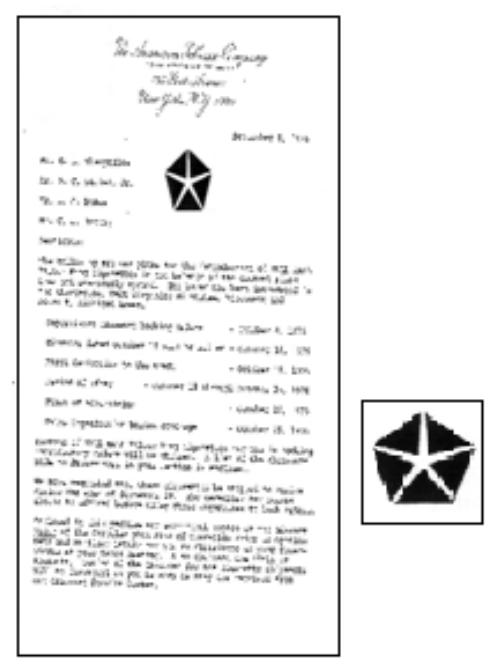

(a) Document-1

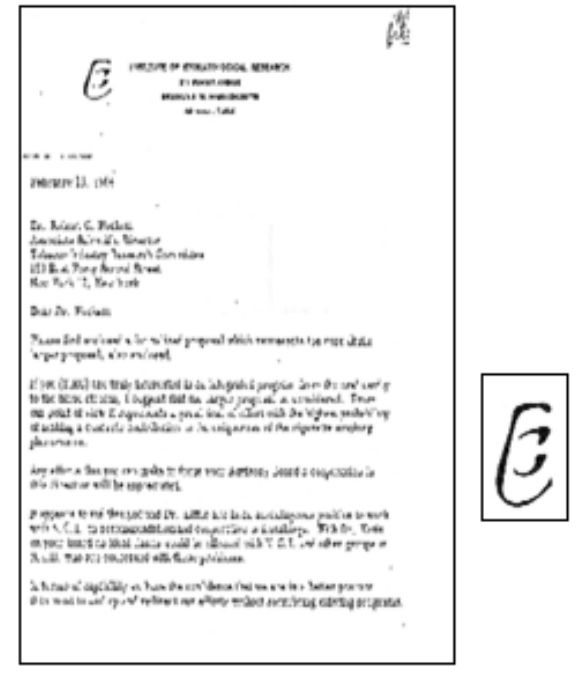

(b) Document-2

Figure 3. Samples of documents and logo extraction from documents 


\section{CONCLUSION}

This paper presents a simple logo extraction method based on morphological operations. The method works relatively faster and efficiently with few limitations. These limitations can be overcome by further improvement in preprocessing techniques.

\section{REFERENCES}

[1] The University of Maryland logo database, http://lampsrv01.umiacs.umd.edu/projdb/project.php?id=47.

[2] Seiden S., M. Dillencourt, S. Irani, R. Borrey and T. Murphy, "Logo detection in document images," in Proc. Int. conf. Imaging science, Sys, and Tech, pp. 446-449, (1997).

[3] Hsieh I.S. and K.C. Fan, "Multiple Classifiers for Color Flag and Trademark Image Retrieval", IEEE Transactions on Image Processing, 10(6): pp. 938-950, (2001).

[4] Yin P.Y and C.C. Yeh, "Content-based retrieval from trademark databases", Pattern Recognition Letters, 23: pp. 113-126, (2002).

[5] Pham T., "Unconstrained logo detection in document images," Pattern recognition, 36: pp. 30233025, (2003).

[6] Hung M.H., C.H. Hsieh, C.M. Kuo, "Similarity retrieval of shape images based on database classification”, Journal Vis. Communication and Image Representation, 17: pp. 970-985, (2006).

[7] Zhu G. and D. Doermann, "Automatic document logo detection," In conference on document analysis and recognition, pp. 864-868, (2007).

[8] Wang H. Y. and Chen, "Logo detection in document images based on boundary extension of feature rectangles," IEEE computer society,10th Int'l conference on document analysis and recognition, pp. 1335-1339, (2009).

[9] Zhu, G. and D. Doermann, "Logo matching for document image retrieval", Int'l Conference on Document Analysis and Recognition, pp. 606-610, (2009).

[10]M.S.Shirdhonkar and Manesh Kokare, "Automatic Logo Detection in Document Images", in proceeding of 2010 IEEE International Conference on Computational Intelligence and Computer Research, held at TCE, Coimbatore, pp. 905-907, (2010).

[11] Sina Hassanzadeh and Hossein Pourghassem, "A Novel Logo Detection and Recognition Framework for Separated Part Logos in Document Images", Australian Journal of Basic and Applied Sciences, 5(9): pp. 936-946, (2011).

[12] Rajib kumar Jha, Prabir Kumar Biswas, B.N.Chattarji, "Logo extraction using combined discrete wavelet transform and dynamic stochastic resonance", International Journal of Image and Graphics, Vol. 1, 2013.

[13] Rajib Kumar Jha, Rajlaxmi Chohan, Kiyoharu Aizawa, "Dynamic stochastic resonance-based improved logo extraction in discrete cosine transform domain”, Journal of Computers and Electrical Engineering, Vol. 40, Issue 6, pp. 1917-1929, 2014.

\section{AUTHORS}

Dr. M.S.Shirdhonkar has received B.E (CSE), M.E (CSE) from Shivaji University, Kolhapur and his Doctorate from Swami Ramanand Teerth, Marathwada University, Nanded, Maharashtra. He has 16 years of experience. His area of interest is Image processing, Document image retrieval and analysis and Pattern recognition. He has published more than 19 papers in national and international conferences and journals

Mr. Umesh D. Dixit has received B.E (E\&C) and M.Tech (CSE) from Visvesvaraya Technological University, Belagavi. He is having an experience of more than 12 years and his area of interest include Image processing, Document image processing, Embedded systems. Currently pursuing Ph.D under Visvesvaraya Technological University, Belagavi, Karnataka.

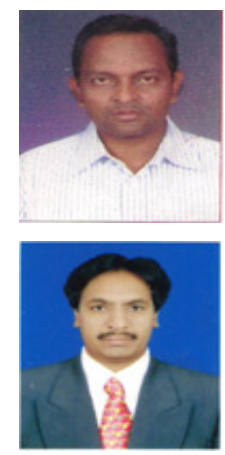

